Bull. Chem. Soc. Ethiop. 2012, 26(2), 257-265.

Printed in Ethiopia

DOI: http://dx.doi.org/10.4314/bcse.v26i2.9

ISSN 1011-3924

(c) 2012 Chemical Society of Ethiopia

\title{
MICROWAVE-ASSISTED SYNTHESIS AND EVALUATION OF ANTIBACTERIAL ACTIVITY OF 2,2'-(NAPHTHALENE-2,7-DIYLBIS(OXY))BIS(N'-SUBSTITUTED ACETOHYDRAZIDE) DERIVATIVES
}

\author{
K. Venkatesan, V.S.V. Satyanarayana, A. Sivakumar* \\ Chemistry Division, School of Advanced Sciences, VIT University, Vellore - 632 014, India
}

(Received September 16, 2011; revised March 9, 2012)

\begin{abstract}
A series of Schiff base of 2,2'-(naphthalene-2,7-diylbis(oxy))bis( $\mathrm{N}^{\prime}$-substituted acetohydrazide) (4a-m) derivatives has been synthesized by the acid catalyzed condensation of aryl/hetero aromatic aldehydes with 2,2'-(naphthalene-2,7-diylbis(oxy))diacetohydrazide (3) under microwave irradiation and conventional method for comparison. The structures of all the newly synthesized compounds have been characterized by IR, ${ }^{1} \mathrm{H}$ NMR, ${ }^{13} \mathrm{C}$ NMR and Mass spectra. All the synthesized compounds have been screened for their in vitro antibacterial activity.
\end{abstract}

KEY WORDS: 2,2'-(Naphthalene-2,7-diylbis(oxy))diacetohydrazide, Schiff base, Microwave irradiation, Spectral characterization, Antibacterial activity

\section{INTRODUCTION}

The chemistry of the carbon-nitrogen double bond plays a vital role in the advancement of synthetic organic chemistry. Schiff bases are usually synthesized by the condensation of primary amines with compounds containing active carbonyl groups. Schiff bases are an important class of compounds in medicine and pharmaceutics. They show biological applications including antibacterial [1-6], antifungal [3-6], antitumor [7, 8], antioxidant [9, 10], anti-inflammatory [11], antihypertensive [12], anti-HIV [13], antifilarial [14], anticonvulsant [15], herbicidal, insecticidal, schistosomicidal and anthelmintic activities [16]. Schiff bases are used as protective agents in natural rubber [17]. Recently there has been a considerable interest in the chemistry of hydrazine and hydrazone compounds because of their potential pharmacological applications [18]. The remarkable reactivity of acid hydrazides $\mathrm{R}-\mathrm{CONHNH}_{2}$ [19] and the biological activity associated with their corresponding hydrazones attributed to the presence of the active pharmacophore (-CONH-N=CH-). Hence many hydrazone compounds containing this active moiety are known to exhibit good anticancer bioactivities according to the literature [20]. Some of the Schiff bases complex combinations with metals are used as insecticides, fungicides, herbicides [21].

Microwave-assisted organic synthesis (MAOS) has been known since 1986 [22]. This "nonconventional" synthetic method has shown broad applications as a very efficient way to accelerate the course of many organic reactions, producing high yields and higher selectivity, lower quantities of side products and consequently, easier work-up procedures and purification of the products. MAOS is considered as "green" technology, principally since many organic reactions can be carried out in solvent-free conditions [23]. In recent years, researchers have applied microwave as a tool in order to reduce reaction time, avoid side products, increase yield and simplify the course of reaction for combinatorial chemistry [24]. Solvent-free reactions are of interest not only from an ecological point of view but in many cases they also offer considerable advantages in terms of yield, selectivity and simplicity.

*Corresponding author. E-mail: svkmr7627@gmail.com 
In many cases reactions that normally require many hours at reflux temperature under normal synthetic conditions can be completed within several minutes or even seconds in a microwave oven. Recent simplification of microwave organic reaction enhancement (MORE) technique has increased safety and practical utility of the microwave oven for their use in organic laboratories without any modification.

Hence, in this paper, we are reporting eco-friendly synthesis of some new Schiff bases by condensing 2,2'-(naphthalene-2,7-diylbis(oxy))diacetohydrazide with aryl/hetero aromatic aldehydes using microwave irradiation technique and the yields are compared with traditional method and their characterization carried out using IR, ${ }^{1} \mathrm{H} \mathrm{NMR},{ }^{13} \mathrm{CNMR}$ and Mass spectra. All the synthesized compounds have been screened for their antibacterial activity.

\section{EXPERIMENTAL}

\section{Screening of antibacterial activity}

The newly synthesized Schiff base compounds (4a-m) were screened for their antibacterial activity against Escherichia coli (ATTC-25922) (E. coli), Staphylococcus aureus (ATTC25923) (S. aureus) and Pseudomonas aeruginosa (ATCC-27853) (P. aeruginosa) bacterial strains by agar well diffusion method in Muller Hinton agar medium. Twenty milliliters of agar media was poured into each Petri dish and plates were dried by placing in an incubator at $37^{\circ} \mathrm{C}$ for an hour. Using a punch, wells were made on these seeded agar plates and concentrations of the test compounds in dimethylsulfoxide (DMSO) were added into each labelled well. A control was also prepared for the plates in the same way using solvent DMSO. All the plates were incubated at $37{ }^{\circ} \mathrm{C}$ for $24 \mathrm{hrs}$ for incubation. The degree of effectiveness was measured by determining the diameters of the zone of inhibition caused by the compounds. Activity of each compound was compared with standard drug tetracycline which is available in the market.

\section{General}

All reagents were purchased from Aldrich, SD Fine Chemicals and Qualigens and used without further purification. For the microwave irradiation experiments described below a conventional (unmodified) household microwave oven equipped with a turntable was used (LG, MG-395 WA, $760 \mathrm{~W}$ ) and operating at $2450 \mathrm{MHz}$. The melting points were determined by open capillaries and are uncorrected. Infrared (IR) spectra were recorded at room temperature from $4000 \mathrm{~cm}^{-1}$ to $400 \mathrm{~cm}^{-1}$ with $\mathrm{KBr}$ pellets at a resolution of $4 \mathrm{~cm}^{-1}$, using Avatar 330 equipped with DTGS detector. ${ }^{1} \mathrm{H}$ NMR and ${ }^{13} \mathrm{C}$ NMR spectra were recorded on a Bruker AV-500 MHz, spectrometer in DMSO- $d_{6}, \mathrm{CDCl}_{3}$ using TMS as internal standard and the values are expressed in ppm. Mass spectra were obtained by using HRMS.

Procedure for the preparation of compound diethyl 2,2'-[naphthalene-2,7-diylbis(oxy)]di acetate (2)

To a solution of 2,7-dihyroxy naphthalene (1) (1.0 molar equiv.) in dry DMF, anhydrous potassium carbonate (2.0 molar equiv.) and ethyl chloroacetate (2.0 molar equiv.) were added. The resultant mixture was stirred at $80{ }^{\circ} \mathrm{C}$ for $16 \mathrm{~h}$, cooled and then it was poured into a large amount of water. The solid separated was filtered and washed with excess of water. The crude product was recrystallized from ethanol. Yield of the product was 81-82\% [lit. yield 40\%] [25]. M.p. 92-94 ${ }^{\circ} \mathrm{C}$; IR (KBr) v $v_{\max }$ : 3258, 2917, 1760, 1654, 1628, 1518, 1384, 1204, 1173, 1076, $1024 \mathrm{~cm}^{-1}$; ${ }^{1} \mathrm{H}$ NMR $\left(500 \mathrm{MHz}, \mathrm{CDCl}_{3}, \mathrm{ppm}\right), \delta_{\mathrm{H}}=7.71(\mathrm{~d}, 2 \mathrm{H}, J=9.0 \mathrm{~Hz}, \mathrm{C}-4,5 \mathrm{ArH}$ of naphthalene ring), $7.12(\mathrm{dd}, 2 \mathrm{H}, J=2.5$ and $9.0 \mathrm{~Hz}, \mathrm{C}-3,6 \mathrm{ArH}$ of naphthalene ring), 6.99 (d, 
$2 \mathrm{H}, J=2.5 \mathrm{~Hz}, \mathrm{C}-1,8 \mathrm{ArH}$ of naphthalene ring), $4.73\left(\mathrm{~s}, 4 \mathrm{H}, \mathrm{OCH}_{2}\right), 4.31(\mathrm{q}, 4 \mathrm{H}, J=7.25 \mathrm{~Hz}$, $\left.\mathrm{COOCH}_{2}\right), 1.33\left(\mathrm{t}, 6 \mathrm{H}, J=7.25 \mathrm{~Hz}, \mathrm{CH}_{3}\right) ;{ }^{13} \mathrm{C} \mathrm{NMR}\left(125.757 \mathrm{MHz}, \mathrm{CDCl}_{3}, \mathrm{ppm}\right), \delta_{\mathrm{C}}=168.84$, 156.51, 135.36, 129.47, 125.26, 116.48, 106.74, 65.51, 61.43, 14.17; HRMS (EI): $\left.\mathrm{m} / z . \mathrm{M}^{+}\right]$ calcd. for $\mathrm{C}_{18} \mathrm{H}_{20} \mathrm{O}_{6}: 332.1260$; found: 332.1254

Procedure for the preparation of compound 2,2'-[naphthalene-2,7-diylbis(oxy)]diaceto hydrazide (3)

Compound $2(0.01 \mathrm{~mole})$ in ethanol $(20 \mathrm{~mL})$ were stirred at room temperature for $20 \mathrm{~min}$. To this mixture hydrazine hydrate $(0.03 \mathrm{~mol})$ was added and stirring continued for 15 more min. The resultant mixture was refluxed for $6 \mathrm{~h}$ and the separated solid was filtered using a sintered glass funnel. The residue was dried and then desiccated to afford a crystalline powder. The powder was recrystallized from chloroform/methanol and gave half white solid. Yield of the product was $88-90 \%$. M.p. $232-234{ }^{\circ} \mathrm{C}$; IR (KBr) $v_{\max }$ : 3311, 3206, 3029, 2923, 1699, 1622, $1471,1385,1212,1174,1060 \mathrm{~cm}^{-1} ;{ }^{1} \mathrm{H}$ NMR $\left(500 \mathrm{MHz}, \mathrm{DMSO}-d_{6}, \mathrm{ppm}\right), \delta_{\mathrm{H}}=9.41(\mathrm{~s}, 2 \mathrm{H}$, $\mathrm{NH}), 7.76(\mathrm{~d}, 2 \mathrm{H}, J=9.0 \mathrm{~Hz}, \mathrm{C}-4,5 \mathrm{ArH}$ of naphthalene ring), 7.04-7.16 (m, 4H, C-1,3,6,8 $\mathrm{ArH}$ of naphthalene ring), 4.86 and $4.96\left(2 \mathrm{~s}, 4 \mathrm{H}, \mathrm{OCH}_{2}\right), 4.58\left(\mathrm{~s}, 4 \mathrm{H}, \mathrm{NH}_{2}\right) ;{ }^{13} \mathrm{CNMR}(125.757$ $\left.\mathrm{MHz}, \mathrm{DMSO}-d_{6}, \mathrm{ppm}\right), \delta_{\mathrm{C}}=169.15,167.03,156.76,156.54,135.72,129.68,129.54,124.81$, 116.84, 116.76, 107.13, 107.01, 66.72, 65.17; HRMS (EI): $\mathrm{m} / z\left[\mathrm{M}^{+}\right]$calcd. for $\mathrm{C}_{14} \mathrm{H}_{16} \mathrm{~N}_{4} \mathrm{O}_{4}$ : 304.1172; found: 304.1169 .

General procedure for the preparation of compound 2,2'-[Naphthalene-2,7-diylbis(oxy)]bis [N'-substituted acetohydrazide] $(4 a-m)$

Method A. A mixture of compound 2,2'-(naphthalene-2,7-diylbis(oxy))diacetohydrazide 3 (0.01 $\mathrm{mol})$ in chloroform/methanol $(1: 1)$ mixture $(30 \mathrm{~mL})$, aryl/hetero aromatic aldehyde $(0.02 \mathrm{~mole})$ and $1 \mathrm{~mL}$ of glacial acetic acid was refluxed for $4-5 \mathrm{~h}$. The mixture was allowed to cool; the solid separated was filtered, washed with excess of methanol and then dried at room temperature.

Method B. A mixture of 2,2'-(naphthalene-2,7-diylbis(oxy))diacetohydrazide 3 (0.01 mol), aryl/hetero aromatic aldehyde $(0.02 \mathrm{~mol})$ and catalytic amount of glacial acetic acid were taken in DMSO $(2 \mathrm{~mL})$ in a beaker and then the reaction mixture was subjected to microwave irradiation at an interval of $1 \mathrm{~min}$ at $180 \mathrm{~W}$ for about 1-2 min; progress of the reaction was monitored by TLC. After the completion of the reaction, the obtained product was poured into ice cold water stirred well and the solid separated was filtered, washed with excess of methanol and then dried at room temperature.

2,2'-[Naphthalene-2,7-diylbis(oxy)]bis[N'-benzylideneacetohydrazide] (4a). M.p. 199-201 ${ }^{\circ} \mathrm{C}$; IR (KBr) $v_{\max }: 3432,3091,2925,1691,1629,1399,1259,1070 \mathrm{~cm}^{-1} ;{ }^{1} \mathrm{H}$ NMR $(500 \mathrm{MHz}$, DMSO- $d 6, \mathrm{ppm}), \delta_{\mathrm{H}}=11.61(\mathrm{t}, 2 \mathrm{H}, J=8.25 \mathrm{~Hz}, \mathrm{NH}), 8.34(\mathrm{~d}, 1 \mathrm{H}, J=22.0 \mathrm{~Hz}, \mathrm{~N}=\mathrm{CH}), 8.03$ $(\mathrm{d}, 1 \mathrm{H}, J=10.5 \mathrm{~Hz}, \mathrm{~N}=\mathrm{CH}), 7.69-7.83(\mathrm{~m}, 6 \mathrm{H}, o-\mathrm{ArH}$ of bezylidene ring and C-4,5 ArH of naphthalene ring), 7.41-7.53 (m, 6H, m,p- ArH of bezylidene ring), 7.05-7.26 (m, 4H, C-1,8 and C-3,6 ArH of naphthalene ring), $5.25(\mathrm{~d}, 2 \mathrm{H}, J=11.0 \mathrm{~Hz}, \mathrm{OCH} 2), 4.77(\mathrm{~d}, 2 \mathrm{H}, J=13.0 \mathrm{~Hz}$, $\left.\mathrm{OCH}_{2}\right) ;{ }^{13} \mathrm{C}$ NMR $\left(125.757 \mathrm{MHz}, \mathrm{DMSO}-d_{6}, \mathrm{ppm}\right), \delta_{\mathrm{C}}=169.36,164.64,157.24,157.15$, $156.81,156.70,148.47,144.34,144.26,135.94,135.85,134.46,130.64,130.38,129.57$, $129.51,129.41,129.39,129.29,129.24,128.83,127.60,127.42,124.74,124.55,116.79$, 116.55, 107.17, 106.99, 106.91, 67.07, 65.25; HRMS (EI): $\mathrm{m} / z$. $[\mathrm{M}+]$ calcd. for $\mathrm{C}_{28} \mathrm{H}_{24} \mathrm{~N}_{4} \mathrm{O}_{4}$ : 480.1798; found: 480.1784 . 
2,2'-[Naphthalene-2,7-diylbis(oxy)]bis[N'-(4-chlorobenzylidene)acetohydrazide] (4b). M.p. 236-238 ${ }^{\circ} \mathrm{C}$; IR (KBr) v $v_{\max }$ : 3428, 3091, 2979, 1689, 1630, 1514, 1402, 1308, 1206, 1178, 1088 $\mathrm{cm}^{-1} ;{ }^{1} \mathrm{H}$ NMR $\left(500 \mathrm{MHz}, \mathrm{DMSO}-d_{6}, \mathrm{ppm}\right), \delta_{\mathrm{H}}=11.67(\mathrm{~d}, 2 \mathrm{H}, J=12.0 \mathrm{~Hz}, \mathrm{NH}), 8.33(\mathrm{~d}, 1 \mathrm{H}, J$ $=14.0 \mathrm{~Hz}, \mathrm{~N}=\mathrm{CH}), 8.02(\mathrm{~d}, 1 \mathrm{H}, J=9.5 \mathrm{~Hz}, \mathrm{~N}=\mathrm{CH}), 7.70-7.71(\mathrm{~m}, 6 \mathrm{H}, o-$ ArH of bezylidene ring and $\mathrm{C}-4,5 \mathrm{ArH}$ of naphthalene ring), 7.47-7.60 (m, 4H, $m$-ArH of bezylidene ring), 7.04$7.24\left(\mathrm{~m}, 4 \mathrm{H}, \mathrm{C}-1,8\right.$ and $\mathrm{C}-3,6 \mathrm{ArH}$ of naphthalene ring), $5.24\left(\mathrm{~d}, 2 \mathrm{H}, J=11.5 \mathrm{~Hz}, \mathrm{OCH}_{2}\right), 4.77$ (d, $2 \mathrm{H}, J=12.0 \mathrm{~Hz}, \mathrm{OCH}_{2}$ ); HRMS (EI): $\mathrm{m} / z$, $\left.\mathrm{M}+\right]$ calcd. for $\mathrm{C}_{28} \mathrm{H}_{22} \mathrm{Cl}_{2} \mathrm{~N}_{4} \mathrm{O}_{4}:$ 548.1018; found: 548.1015 .

2,2'-[Naphthalene-2,7-diylbis(oxy)]bis[N'-(4-methoxybenzylidene)acetohydrazide] (4c). M.p. $189-191{ }^{\circ} \mathrm{C}$; IR (KBr) $v_{\max } 3424,3059,2913,1678,1605,1540,1509,1445,1384,1253,1170$, $1084 \mathrm{~cm}^{-1} ;{ }^{1} \mathrm{H}$ NMR $\left(500 \mathrm{MHz}\right.$, DMSO- $\left.d_{6}, \mathrm{ppm}\right), \delta_{\mathrm{H}}=11.46(\mathrm{t}, 2 \mathrm{H}, J=12.75 \mathrm{~Hz}, \mathrm{NH}), 8.29(\mathrm{~s}$, $1 \mathrm{H}, \mathrm{N}=\mathrm{CH}), 7.97(\mathrm{~d}, 1 \mathrm{H}, J=10.5 \mathrm{~Hz}, \mathrm{~N}=\mathrm{CH}), 7.74-7.82(\mathrm{~m}, 2 \mathrm{H}, \mathrm{C}-4,5 \mathrm{ArH}$ of naphthalene ring), 7.62-7.68 (m, 4H, o-ArH of bezylidene ring), 7.04-7.25 (m, 4H, C-1,8 and C-3,6 ArH of naphthalene ring), 6.97-7.01 (m, 4H, $m$-ArH of bezylidene ring), $5.21(\mathrm{~d}, 2 \mathrm{H}, J=12.0 \mathrm{~Hz}$, $\left.\mathrm{OCH}_{2}\right), 4.74\left(\mathrm{~d}, 2 \mathrm{H}, J=13.5 \mathrm{~Hz}, \mathrm{OCH}_{2}\right) ; 3.79$ (d, 6H, $\left.J=8.5 \mathrm{~Hz}, \mathrm{OCH} 3\right)$; HRMS (EI): $\mathrm{m} / \mathrm{z}$ [M+] calcd. For $\mathrm{C}_{30} \mathrm{H}_{28} \mathrm{~N}_{4} \mathrm{O}_{6}: 540.2009$; found: 540.2004.

2,2'-[Naphthalene-2,7-diylbis(oxy)]bis[N'-(2,4-dichlorobenzylidene)acetohydrazide] (4d). M.p. 230-232 ${ }^{\circ} \mathrm{C}$; IR (KBr) $v_{\max }: 3432,3091,2925,1691,1629,1399,1259,1070 \mathrm{~cm}-1 ;{ }^{1} \mathrm{H}$ NMR (500 MHz, DMSO- $\left.d_{6}, \mathrm{ppm}\right), \delta_{\mathrm{H}}=11.83$ and $11.92(2 \mathrm{~d}, 2 \mathrm{H}, J=9.0$ and $9.5 \mathrm{~Hz}, \mathrm{NH}), 8.36 \&$ $8.71(\mathrm{~d}$ and s, $1 \mathrm{H}, J=10.0 \mathrm{~Hz}, \mathrm{~N}=\mathrm{CH}), 7.96$ and $8.05(\mathrm{~d} \& \mathrm{t}, 1 \mathrm{H}, J=8.5$ and $9.0 \mathrm{~Hz}, \mathrm{~N}=\mathrm{CH})$, 7.70-7.83 (m, $4 \mathrm{H}, o-\mathrm{ArH}$ of bezylidene ring and $\mathrm{C}-4,5 \mathrm{ArH}$ of naphthalene ring), 7.44-7.52 (m, $2 \mathrm{H}, m$-ArH of bezylidene ring), 7.05-7.26 (m, 6H, C-1,8 and $\mathrm{C}-3,6 \mathrm{ArH}$ of naphthalene ring and $m$-ArH of bezylidene ring), $5.26\left(\mathrm{~d}, 2 \mathrm{H}, J=12.0 \mathrm{~Hz}, \mathrm{OCH}_{2}\right), 4.79\left(\mathrm{~d}, 2 \mathrm{H}, J=12.0 \mathrm{~Hz}, \mathrm{OCH}_{2}\right)$; HRMS (EI): $m / z$ [M+] calcd. for $\mathrm{C}_{28} \mathrm{H}_{20} \mathrm{Cl}_{4} \mathrm{~N}_{4} \mathrm{O}_{4}$ : 618.0209; found: 618.0209.

2,2'-[Naphthalene-2,7-diylbis(oxy)]bis[N'-(4-(dimethylamino)benzylidene)acetohydrazide] (4e). M.p. 220-222 ${ }^{\circ} \mathrm{C}$; IR (KBr) $v_{\max }: 3443,2926,1664,1604,1520,1368,1213,1169,1068$ $\mathrm{cm}^{-1} ;{ }^{1} \mathrm{H}$ NMR $\left(500 \mathrm{MHz}, \mathrm{DMSO}-d_{6}, \mathrm{ppm}\right), \delta_{\mathrm{H}}=11.30$ (split peak, $\left.2 \mathrm{H}, \mathrm{NH}\right), 8.19(\mathrm{~s}, 1 \mathrm{H}$, $\mathrm{N}=\mathrm{CH}), 7.90(\mathrm{~d}, 1 \mathrm{H}, J=11.5 \mathrm{~Hz}, \mathrm{~N}=\mathrm{CH}), 7.64-7.81(\mathrm{~m}, 2 \mathrm{H}, \mathrm{C}-4,5 \mathrm{ArH}$ of naphthalene ring), 7.49-7.54 (m, 4H, o-ArH of bezylidene ring), 7.03-7.25 (m, 4H, C-1,8 and C-3,6 ArH of naphthalene ring), 6.70-6.77 (m, $4 \mathrm{H}, m$-ArH of bezylidene ring), $5.19(\mathrm{~d}, 2 \mathrm{H}, J=12.0 \mathrm{~Hz}$, OCH2), 4.71 (d, 2H, $J=14.5 \mathrm{~Hz}, \mathrm{OCH} 2), 2.98$ (q, 12H, $J=19.0 \mathrm{~Hz}, \mathrm{CH} 3) ;{ }^{13} \mathrm{C}$ NMR $(125.757$ MHz, DMSO- $\left.d_{6}, \mathrm{ppm}\right), \delta_{\mathrm{C}}=168.74,168.72,163.91,160.26,157.29,157.21,156.86,156.74$, $152.05,151.89,151.84,149.31,145.18,145.08,135.97,135.86,129.95,129.64,129.53$, $129.48,129.37,128.94,128.68,124.69,124.50,121.82,116.76,116.48,112.24,112.16$, 107.15, 106.94, 67.13, 65.27; HRMS (EI): $\mathrm{m} / z . \mathrm{M}+]$ calcd. for $\mathrm{C}_{32} \mathrm{H}_{34} \mathrm{~N}_{6} \mathrm{O}_{4}: 566.2642$; found: 566.2640 .

2,2'-[Naphthalene-2,7-diylbis(oxy)]bis[N'-(4-ethoxy-3-methoxybenzylidene)acetohydrazide] (4f). M.p. 169-171 ${ }^{\circ} \mathrm{C}$; IR (KBr) $v_{\max }: 3442$, 3061, 2927, 1679, 1634, 1513, 1466, 1391, 1266, $1139,1080 \mathrm{~cm}-1 ;{ }^{1} \mathrm{H}$ NMR (500 MHz, DMSO- $\left.d_{6}, \mathrm{ppm}\right), \delta_{\mathrm{H}}=11.48(\mathrm{q}, 2 \mathrm{H}, J=16.0 \mathrm{~Hz}, \mathrm{NH})$, $8.26(\mathrm{~s}, 1 \mathrm{H}, \mathrm{N}=\mathrm{CH}), 7.94(\mathrm{~d}, 1 \mathrm{H}, J=12.0 \mathrm{~Hz}, \mathrm{~N}=\mathrm{CH}), 7.74-7.82(\mathrm{~m}, 2 \mathrm{H}, \mathrm{C}-4,5$ ArH of naphthalene ring), 7.29-7.34 (m, 2H, $o$-ArH of bezylidene ring), 7.04-7.23 (m, 6H, C-1,3,6,8 ArH of naphthalene ring and $o$-ArH of bezylidene ring), $6.95-7.00(\mathrm{~m}, 2 \mathrm{H}, m$ - $\mathrm{ArH}$ of bezylidene ring), $5.24\left(\mathrm{~d}, 2 \mathrm{H}, J=14.0 \mathrm{~Hz}, \mathrm{OCH}_{2}\right), 4.74\left(\mathrm{~d}, 2 \mathrm{H}, J=15.0 \mathrm{~Hz}, \mathrm{OCH}_{2}\right), 4.01-4.07(\mathrm{~m}, 4 \mathrm{H}$, $\mathrm{OCH}_{2}$ of ethoxy group), $3.78\left(\mathrm{t}, 6 \mathrm{H}, J=5.0\right.$ and $\left.10.5 \mathrm{~Hz}, \mathrm{OCH}_{3}\right), 1.33(\mathrm{q}, 6 \mathrm{H}, J=6.75 \mathrm{~Hz}$, $\mathrm{CH}_{3}$ ); HRMS (EI): $\mathrm{m} / z$ [M+] calcd. For $\mathrm{C}_{34} \mathrm{H}_{36} \mathrm{~N}_{4} \mathrm{O}_{8}$ : 628.2533; found: 628.0453. 
2,2'-[Naphthalene-2,7-diylbis(oxy)]bis[N'-(3-nitrobenzylidene)acetohydrazide] (4g). M.p. 251$253{ }^{\circ} \mathrm{C}$; IR (KBr) v $v_{\max }: 3429,3190,3043,2920,1676,1628,1385,1255,1169,1067 \mathrm{~cm}-1 ;{ }^{1} \mathrm{H}$ NMR (500 MHz,DMSO- $\left.d_{6}, \mathrm{ppm}\right), \delta_{\mathrm{H}}=11.85(\mathrm{t}, 2 \mathrm{H}, J=16.0 \mathrm{~Hz}, \mathrm{NH}), 8.46-8.51$ (split peak, $2 \mathrm{H}, \mathrm{N}=\mathrm{CH}), 8.12-8.24(\mathrm{~m}, 6 \mathrm{H}, o$ - and $p$-ArH of bezylidene ring), 7.67-7.82 (m, $4 \mathrm{H}, m$-ArH of bezylidene ring and C-4,5 ArH of naphthalene ring), 7.06-7.23 (m, 4H, C-1,8 and C-3,6 ArH of naphthalene ring), $5.30\left(\mathrm{~d}, 2 \mathrm{H}, J=12.5 \mathrm{~Hz}, \mathrm{OCH}_{2}\right), 4.80\left(\mathrm{~d}, 2 \mathrm{H}, J=12.5 \mathrm{~Hz}, \mathrm{OCH}_{2}\right)$; HRMS (EI): $m / z,[\mathrm{M}+]$ calcd. for $\mathrm{C}_{28} \mathrm{H}_{22} \mathrm{~N}_{6} \mathrm{O}_{8}$ : 570.1499; found: 570.1495 .

2,2'-[Naphthalene-2,7-diylbis(oxy)]bis[N'-(3-ethoxy-4-ydroxybenzylidene)acetohydrazide] (4h). M.p. 210-212 ${ }^{\circ} \mathrm{C}$; IR (KBr) $v_{\max }: 3369,3070,2989,1668,1598,1511,1438,1394,1284$, $1167,1070 \mathrm{~cm}^{-1} ;{ }^{1} \mathrm{H}$ NMR (500 MHz, DMSO- $\left.d_{6}, \mathrm{ppm}\right), \delta_{\mathrm{H}}=11.42(\mathrm{t}, 2 \mathrm{H}, J=16.5 \mathrm{~Hz}, \mathrm{NH})$, $9.48(\mathrm{~d}, 1 \mathrm{H}, J=3.0 \mathrm{~Hz}, \mathrm{OH}), 9.41(\mathrm{~d}, 1 \mathrm{H}, J=11.0 \mathrm{~Hz}, \mathrm{OH}), 8.21(\mathrm{~s}, 1 \mathrm{H}, \mathrm{N}=\mathrm{CH}), 7.89$ (d, 2H, $J$ $=11.0 \mathrm{~Hz}, \mathrm{~N}=\mathrm{CH}), 7.74-7.80(\mathrm{~m}, 2 \mathrm{H}, \mathrm{C}-4,5 \mathrm{ArH}$ of naphthalene ring), 7.05-7.28 (m, $8 \mathrm{H}, o-$ ArH of bezylidene ring and C-1,8 and C-3,6 ArH of naphthalene ring), 6.81-6.85 (q, 2H, $J=$ $8.25 \mathrm{~Hz}, m$-ArH of bezylidene ring), $5.21\left(\mathrm{~d}, 2 \mathrm{H}, J=13.0 \mathrm{~Hz}, \mathrm{OCH}_{2}\right), 4.73(\mathrm{~d}, 2 \mathrm{H}, J=13.5 \mathrm{~Hz}$, $\left.\mathrm{OCH}_{2}\right), 4.00-4.05\left(\mathrm{~m}, 4 \mathrm{H}, \mathrm{OCH}_{2}\right.$ of ethoxy), 1.28-1.36 (m, $\left.6 \mathrm{H}, \mathrm{CH}_{3}\right)$; HRMS (EI): $\mathrm{m} / z$ [M+] calcd. for $\mathrm{C}_{32} \mathrm{H}_{32} \mathrm{~N}_{4} \mathrm{O}_{8}$ : 600.2220;found: 600.2219 .

2,2'-[Naphthalene-2,7-diylbis(oxy)]bis[N'-(4-hydroxy-3-methoxybenzylidene) acetohydrazide] (4i). M.p. 216-218 ${ }^{\circ} \mathrm{C}$; IR (KBr) $v_{\max }$ : 3445, 3048, 2993, 2432, 1661, 1606, 1513, 1437, 1291, $1163,1077,1027 \mathrm{~cm}^{-1} ;{ }^{1} \mathrm{H}$ NMR $\left(500 \mathrm{MHz}, \mathrm{DMSO}-d_{6}, \mathrm{ppm}\right), \delta_{\mathrm{H}}=11.42(\mathrm{t}, 2 \mathrm{H}, J=17.5 \mathrm{~Hz}$, $\mathrm{NH}), 9.51$ (split peak, 2H, OH), $8.23(\mathrm{~s}, 1 \mathrm{H}, \mathrm{N}=\mathrm{CH}), 7.91(\mathrm{~d}, 1 \mathrm{H}, J=9.5 \mathrm{~Hz}, \mathrm{~N}=\mathrm{CH}), 7.74-7.80$ $\left(\mathrm{m}, 2 \mathrm{H}, \mathrm{C}-4,5 \mathrm{ArH}\right.$ of naphthalene ring), 7.04-7.23 (m, 8H, o, $o^{\prime}-\mathrm{ArH}$ of bezylidene ring and $\mathrm{C}$ 1,8 and $\mathrm{C}-3,6 \mathrm{ArH}$ of naphthalene ring), $6.82(\mathrm{t}, 2 \mathrm{H}, J=8.5$ and $8.5 \mathrm{~Hz}, m$-ArH of bezylidene ring), $5.23\left(\mathrm{~d}, 2 \mathrm{H}, J=13.0 \mathrm{~Hz}, \mathrm{OCH}_{2}\right), 4.74\left(\mathrm{~d}, 2 \mathrm{H}, J=13.5 \mathrm{~Hz}, \mathrm{OCH}_{2}\right), 3.79$ (split peak, $6 \mathrm{H}$, $\mathrm{OCH}_{3}$ ); HRMS (EI): $m / z$ [M+] calcd. for $\mathrm{C}_{30} \mathrm{H}_{28} \mathrm{~N}_{4} \mathrm{O}_{8}$ : 572.1907; found: 572.1905 .

2,2'-[Naphthalene-2,7-diylbis(oxy)]bis[N'-(3-methylbenzylidene)acetohydrazide] (4j). M.p. 227-229 ${ }^{\circ} \mathrm{C}$; IR (KBr) $v_{\max }$ : 3417, 3262, 3070, 2989, 1668, 1598, 1438, 1394, 1284, 1167, 1080 $\mathrm{cm}^{-1} ;{ }^{1} \mathrm{H}$ NMR $\left(500 \mathrm{MHz}, \mathrm{DMSO}-d_{6}, \mathrm{ppm}\right), \delta_{\mathrm{H}}=11.58(\mathrm{~d}, 2 \mathrm{H}, J=15.0 \mathrm{~Hz}, \mathrm{NH}), 8.30(\mathrm{~s}, 1 \mathrm{H}$, $\mathrm{N}=\mathrm{CH}), 7.99(\mathrm{~d}, 1 \mathrm{H}, J=12.5 \mathrm{~Hz}, \mathrm{~N}=\mathrm{CH}), 7.75-7.82(\mathrm{~m}, 2 \mathrm{H}, \mathrm{C}-4,5 \mathrm{ArH}$ of naphthalene ring), 7.46-7.55 (m, $4 \mathrm{H}, o$-ArH of bezylidene ring), 7.04-7.34 $(\mathrm{m}, 8 \mathrm{H}, m, p$-ArH of benzylidene ring and $\mathrm{C}-1,8$ and $\mathrm{C}-3,6 \mathrm{ArH}$ of naphthalene ring), $5.24\left(\mathrm{~d}, 2 \mathrm{H}, J=12.5 \mathrm{~Hz}, \mathrm{OCH}_{2}\right), 4.76(\mathrm{~d}, 2 \mathrm{H}, J$ $\left.=14.0 \mathrm{~Hz}, \mathrm{OCH}_{2}\right), 2.33\left(\mathrm{t}, 6 \mathrm{H}, J=7.75 \mathrm{~Hz}, \mathrm{CH}_{3}\right)$; HRMS (EI): $\mathrm{m} / z$ [M+] calcd. for $\mathrm{C}_{30} \mathrm{H}_{28} \mathrm{~N}_{4} \mathrm{O}_{4}$ : 508.2111; found: 508.2108 .

2,2'-[Naphthalene-2,7-diylbis(oxy)]bis[N'-(4-ethoxybenzylidene)acetohydrazide] (4k). M.p. 218-220 ${ }^{\circ} \mathrm{C}$; IR (KBr) $v_{\max }: 3421,3069,2927,1676,1606,1511,1392,1253,1170,1070 \mathrm{~cm}^{-1}$; ${ }^{1} \mathrm{H}$ NMR $\left(500 \mathrm{MHz}, \mathrm{DMSO}-d_{6}, \mathrm{ppm}\right), \delta_{\mathrm{H}}=11.47(\mathrm{q}, 2 \mathrm{H}, J=11.25 \mathrm{~Hz}, \mathrm{NH}), 8.28(\mathrm{~s}, 1 \mathrm{H}$, $\mathrm{N}=\mathrm{CH}), 7.96(\mathrm{~d}, 1 \mathrm{H}, J=10.5 \mathrm{~Hz}, \mathrm{~N}=\mathrm{CH}), 7.74-7.82(\mathrm{~m}, 2 \mathrm{H}, \mathrm{C}-4,5 \mathrm{ArH}$ of naphthalene ring), 7.61-7.67 $(\mathrm{m}, 4 \mathrm{H}, o$-ArH of bezylidene ring), 7.04-7.25 (m, 4H, C-1,8 and C-3,6 ArH of naphthalene ring), 6.94-6.99 (m, $4 \mathrm{H}, m$-ArH of bezylidene ring), $5.21(\mathrm{~d}, 2 \mathrm{H}, J=12.0 \mathrm{~Hz}$, $\left.\mathrm{OCH}_{2}\right), 4.74(\mathrm{~d}, 2 \mathrm{H}, J=13.5 \mathrm{~Hz}, \mathrm{OCH} 2), 4.02-4.079\left(\mathrm{~m}, 4 \mathrm{H}, \mathrm{OCH}_{2}\right.$ of ethoxy group), 1.32-1.35 (m, 6H, CH3); HRMS (EI): $m / z$ [M+] calcd. for $\mathrm{C}_{32} \mathrm{H}_{32} \mathrm{~N}_{4} \mathrm{O}_{6}: 568.2322$; found: 568.2320.

2,2'-[Naphthalene-2,7-diylbis(oxy)]bis[N'-(3-phenylallylidene)acetohydrazide] (4l). M.p. 221$223{ }^{\circ} \mathrm{C}$; IR (KBr) $v_{\max }: 3450,3053,2922,1701,1623,1526,1374,1206,1060 \mathrm{~cm}^{-1} ;{ }^{1} \mathrm{H}$ NMR $\left(500 \mathrm{MHz}, \mathrm{DMSO}-d_{6}, \mathrm{ppm}\right), \delta_{\mathrm{H}}=11.51(\mathrm{t}, 2 \mathrm{H}, J=11.0 \mathrm{~Hz}, \mathrm{NH}), 8.13(\mathrm{~d}, 1 \mathrm{H}, J=4.0 \mathrm{~Hz}$, $\mathrm{N}=\mathrm{CH}), 7.75-7.87(\mathrm{~m}, 3 \mathrm{H}, \mathrm{C}-4,5 \mathrm{ArH}$ of naphthalene ring and $\mathrm{N}=\mathrm{CH}), 7.59-7.62(\mathrm{~m}, 4 \mathrm{H}, o-$ ArH of bezylidene ring), 7.28-7.41 (m, 6H, m,p-ArH of bezylidene ring), 7.05-7.24 (m, 8H, C- 
1,8 and $\mathrm{C}-3,6 \mathrm{ArH}$ of naphthalene ring and $\mathrm{CH}=\mathrm{CH}), 5.14(\mathrm{~d}, 2 \mathrm{H}, J=9.0 \mathrm{~Hz}, \mathrm{OCH} 2), 4.75(\mathrm{~d}$, $\left.2 \mathrm{H}, J=10.0 \mathrm{~Hz}, \mathrm{OCH}_{2}\right)$; $13 \mathrm{C}$ NMR $\left(125.757 \mathrm{MHz}, \mathrm{DMSO}-d_{6}, \mathrm{ppm}\right), \delta_{\mathrm{C}}=168.99,164.48$, $157.17,157.11,150.36,146.87,146.79,139.80,139.48,139.41,136.30,129.56,129.41$, 129.30, 127.58, 127.52, 125.91, 125.46, 116.74, 116.54, 107.12, 106.87, 106.81,67.02, 65.13; HRMS (EI): $m / z[\mathrm{M}+]$ calcd. for $\mathrm{C}_{32} \mathrm{H}_{28} \mathrm{~N}_{4} \mathrm{O}_{4}$ : 532.2111; found: 532.2109.

2,2'-[Naphthalene-2,7-diylbis(oxy)]bis[N'-(thiophen-2-ylmethylene)acetohydrazide] (4m). M.p. $162-164{ }^{\circ} \mathrm{C}$; IR (KBr) $v_{\max }: 3431,3105,2928,1686,1631,1425,1266,1170,1073 \mathrm{~cm}^{-1} ;{ }^{1} \mathrm{H}$ $\operatorname{NMR}\left(500 \mathrm{MHz}, \mathrm{DMSO}-d_{6}, \mathrm{ppm}\right), \delta_{\mathrm{H}}=11.57(\mathrm{q}, 2 \mathrm{H}, J=14.5 \mathrm{~Hz}, \mathrm{NH}), 8.57(\mathrm{~s}, 1 \mathrm{H}, \mathrm{N}=\mathrm{CH})$, $8.21(\mathrm{~d}, 1 \mathrm{H}, J=11.0 \mathrm{~Hz}, \mathrm{~N}=\mathrm{CH}), 7.74-7.82(\mathrm{~m}, 2 \mathrm{H}, \mathrm{C}-4,5 \mathrm{ArH}$ of naphthalene ring), 7.61-7.67 (m, 2H, C-5 ArH of thiophene ring), 7.44-7.46 (m, 2H, C-3 ArH of thiophene ring), 7.03-7.25 $(\mathrm{m}, 6 \mathrm{H}, \mathrm{C}-1,8$ and $\mathrm{C}-3,6 \mathrm{ArH}$ of naphthalene ring and $\mathrm{C}-4 \mathrm{ArH}$ of thiophene ring), $5.14(\mathrm{~d}, 2 \mathrm{H}$, $\left.J=10.5 \mathrm{~Hz}, \mathrm{OCH}_{2}\right), 4.75\left(\mathrm{~d}, 2 \mathrm{H}, J=12.5 \mathrm{~Hz}, \mathrm{OCH}_{2}\right) ;{ }^{13} \mathrm{C} \mathrm{NMR}\left(125.757 \mathrm{MHz}, \mathrm{DMSO} d_{6}\right.$, $\mathrm{ppm}), \delta_{\mathrm{C}}=168.98,164.47,157.20,156.77,156.66,143.63,139.48,139.42,139.29,139.10$, $135.81,131.60,131.01,130.95,129.66,129.56,129.42,129.05,129.00,128.33,124.75$, $116.74,116.56,107.24,107.18,106.92,106.86,67.08,65.02$; HRMS (EI): $m / z[\mathrm{M}+]$ calcd. For $\mathrm{C}_{24} \mathrm{H}_{20} \mathrm{~N}_{4} \mathrm{O}_{4} \mathrm{~S}_{2}$ : 492.0926 ; found: 492.1065 .

\section{RESULTS AND DISCUSSION}

In the present study, diethyl 2,2'-(naphthalene-2,7-diylbis(oxy))diacetate (2) was synthesized from 2,7-dihydroxy naphthalene $\mathbf{1}$ on reacting with ethyl chloroacetate in anhydrous potassium carbonate and dry DMF at $80^{\circ} \mathrm{C}$ for 16 hours resulting in $82 \%$ yield (Scheme 1) [25]. The IR spectrum of compound 2 exhibited absorption frequency at $1760 \mathrm{~cm}^{-1}$ for carbonyl group. In ${ }^{1} \mathrm{H}$ NMR of compound $\mathbf{2}$ the characteristic signals of an ester moiety confirm the presence of ester group in the structure by resonating as quartet and triplet for $-\mathrm{CH}_{2}$ and $-\mathrm{CH}_{3}$ at $\delta 4.31 \mathrm{ppm}$ and $\delta 1.32 \mathrm{ppm}$ respectively, which indicates acetate formation. Treatment of compound 2 with hydrazine hydrate in ethanol and refluxing for 6 hours resulted in the formation of 2,2'(naphthalene-2,7-diylbis(oxy))diacetohydrazide $\mathbf{3}$. The structure of compound $\mathbf{3}$ was confirmed by IR, ${ }^{1} \mathrm{H}$ NMR and Mass spectra. IR spectrum showed the absence of ester stretching frequency, instead it gave band at $1699 \mathrm{~cm}^{-1}$ for carbonyl group and showed two sharp bands in the region of 3311 and $3206 \mathrm{~cm}^{-1}$ due to $-\mathrm{NH}$ and $-\mathrm{NH}_{2}$ groups.
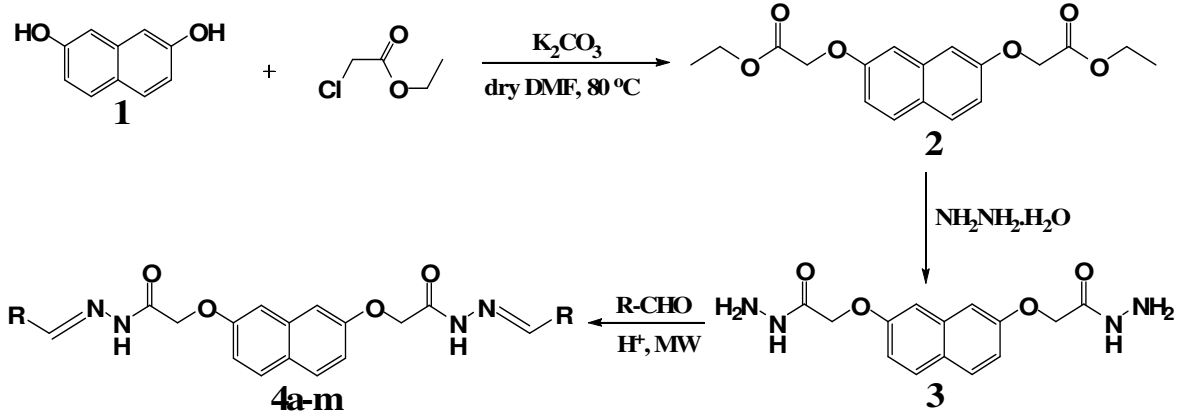

Scheme 1. Synthetic pathway for the preparation of compounds 4a-m. 
Table 1. Data of synthesized Schiff base compounds 4a-m.

\begin{tabular}{|c|c|c|c|c|c|c|}
\hline \multirow{2}{*}{ Entry } & \multirow{2}{*}{$\mathrm{R}$} & \multicolumn{2}{|c|}{ Reaction time (min) } & \multicolumn{2}{|c|}{ Yield $^{\mathrm{a}}(\%)$} & \multirow{2}{*}{ M.p. $\left({ }^{\circ} \mathrm{C}\right)$} \\
\hline & & MW & Conventional & MW & Conventional & \\
\hline $4 \mathbf{a}$ & Phenyl & 1.0 & 180 & 90 & 83 & 199-201 \\
\hline $4 b$ & 4-ClPh & 1.5 & 210 & 88 & 80 & $236-238$ \\
\hline $4 c$ & $4-\mathrm{CH}_{3} \mathrm{OPh}$ & 1.0 & 210 & 87 & 80 & 189-191 \\
\hline $4 d$ & 2,4-ClPh & 1.5 & 240 & 85 & 76 & $230-232$ \\
\hline $4 e$ & $4-\mathrm{N}\left(\mathrm{CH}_{3}\right)_{2} \mathrm{Ph}$ & 1.5 & 240 & 86 & 77 & $220-222$ \\
\hline $4 f$ & 4-OEt,3- $\mathrm{CH}_{3} \mathrm{OPh}$ & 1.5 & 240 & 83 & 75 & $169-171$ \\
\hline $4 \mathrm{~g}$ & $3-\mathrm{NO}_{2} \mathrm{Ph}$ & 2.0 & 240 & 85 & 77 & $251-253$ \\
\hline $4 \mathrm{~h}$ & 3-OEt,4-OHPh & 1.5 & 210 & 84 & 76 & $210-212$ \\
\hline $4 \mathbf{i}$ & $4-\mathrm{OH}, 3-\mathrm{CH}_{3} \mathrm{OPh}$ & 1.5 & 210 & 83 & 75 & $216-218$ \\
\hline $4 \mathbf{j}$ & $3-\mathrm{CH}_{3} \mathrm{Ph}$ & 1.5 & 240 & 86 & 79 & $227-229$ \\
\hline $4 k$ & 4-EtOPh & 1.0 & 210 & 87 & 80 & $218-220$ \\
\hline 41 & Cinnamyl & 2.0 & 240 & 82 & 73 & $221-223$ \\
\hline $4 m$ & 2-Thiophenyl & 1.5 & 210 & 84 & 78 & $162-164$ \\
\hline
\end{tabular}

${ }^{\mathrm{a}}=$ Isolated yield; $\mathrm{MW}=$ Microwave.

${ }^{1} \mathrm{H}$ NMR spectrum of compound $\mathbf{3}$ exhibited no peak corresponding to ester, instead it showed signals at $\delta 9.41 \mathrm{ppm}$ and $\delta 4.58 \mathrm{ppm}$ for $-\mathrm{NH}$ and $-\mathrm{NH}_{2}$ of hydrazide.

Compound 3 was treated with substituted aromatic aldehyde in presence of catalytic amount of acetic acid in (1:1) methanol/chloroform mixture and DMSO under reflux temperature and microwave irradiation to synthesise 2,2'-(naphthalene-2,7-diylbis(oxy))bis(N'-substituted acetohydrazides $\mathbf{4 a - m}$. The IR spectra of the compound $\mathbf{4 a}$ revealed two strong absorption bands at $1629 \mathrm{~cm}^{-1}$ and $1691 \mathrm{~cm}^{-1}$ for $\mathrm{C}=\mathrm{O}$ and $\mathrm{C}=\mathrm{N}$ group respectively. The ${ }^{1} \mathrm{H}$ NMR spectra of compound (4a) displayed two doublets due to methylene protons at $\delta 5.25 \& 4.77 \mathrm{ppm}$ and the $\mathrm{NH}$ group was resonated as triplet at $\delta 11.61 \mathrm{ppm}$. The peaks of naphthalene aromatic and phenyl group protons appeared between $7.05-7.83 \mathrm{ppm}$. The signals belonging to $-\mathrm{N}=\mathrm{CH}$ protons were observed as doublets at $\delta 8.03 \& 8.34 \mathrm{ppm}$, the disappearance of $\mathrm{NH}_{2}$ signal of compound $\mathbf{3}$ in compound $\mathbf{4 a}$ indicates the functionalization of hydrazide to hydrazone. The structures of all the new Schiff base derivatives (4a-m) were confirmed by the spectral characterization using FT-IR, ${ }^{1} \mathrm{H}$ NMR, ${ }^{13} \mathrm{C}$ NMR and mass spectra. Shorter reaction times were observed for the formation of compounds $\mathbf{4 a - m}$ under microwave irradiation rather than the reflux temperature. Reaction time for the formation of compound $\mathbf{4 a}$ is one minute under microwave irradiation and 180 minutes under reflux temperature with slightly improved yields, respectively. Comparative yields of all the synthesized products with respect to reaction time and reflux temperature as well as microwave irradiation and are given in Table 1.

The newly synthesized Schiff base compounds (4a-m) were screened for their antibacterial activity against $E$. coli, $S$. aureus and $P$. aeruginosa bacterial strains by agar well diffusion method in Muller Hinton agar medium. Zone of inhibition was determined for all the newly synthesized compounds and the results are summarized in Table 2. The synthesized compounds 4h and 4i, having 3-ethoxy-4-hydroxybenzylidene and 3-methoxy-4-hydroxybenzylidene ring as substitute $\mathbf{R}$ showed potent activity against S.aureus, E.coli and P.aeruginosa, whereas the compounds $\mathbf{4 d}$ and $\mathbf{4 l}$, having 3-nitro benzylidene and cinnamyl ring as substitute $\mathbf{R}$ showed least activity against $S$.aureus, E.coli and P.aeruginosa. The compounds $4 \mathbf{a}, \mathbf{4 b}, \mathbf{4 c}, \mathbf{4 f}$ and $\mathbf{4 m}$ showed good activity against E.coli, S.aureus and P.aeruginosa. The compound $4 \mathbf{e}$ also showed good activity against E.coli and moderate activity against S.aureus and P.aeruginosa. The above results indicate that the compounds containing electron donating groups show good activity than the compounds containing electron withdrawing groups and the results were depicted in Table 2 . 
Table 2. Antibacterial activities of the Schiff base compounds 4a-m.

\begin{tabular}{|c|c|c|c|}
\hline Compound & \multicolumn{2}{|c|}{ Antibacterial activity ${ }^{\mathrm{a}}(\mathrm{MIC}, \mathrm{mg} / \mathrm{mL})$} \\
\hline & S. aureus & E. coli & Ps. aeruginosa \\
\hline $\mathbf{4 b}$ & $8(250)$ & $8(250)$ & $9(500)$ \\
$\mathbf{4} \mathbf{c}$ & $11(500)$ & $8(250)$ & $8(250)$ \\
$\mathbf{4 d}$ & $8(250)$ & $9(250)$ & $9(250)$ \\
$\mathbf{4 e}$ & $10(750)$ & $11(500)$ & $9(500)$ \\
$\mathbf{4 f}$ & $9(500)$ & $12(500)$ & $9(500)$ \\
$\mathbf{4 g}$ & $9(250)$ & $9(250)$ & $10(250)$ \\
$\mathbf{4 h}$ & $10(500)$ & $8(500)$ & $8(500)$ \\
$\mathbf{4 i}$ & $12(250)$ & $11(250)$ & $8(250)$ \\
$\mathbf{4 j}$ & $19(250)$ & $15(250)$ & $9(250)$ \\
$\mathbf{4 k}$ & $9(500)$ & $10(750)$ & $8(500)$ \\
$\mathbf{4 l}$ & $10(500)$ & $8(500)$ & $9(500)$ \\
$\mathbf{4 m}$ & $11(750)$ & $8(500)$ & $10(500)$ \\
Tetracycline & $9(250)$ & $9(250)$ & $9(500)$ \\
& $14(250)$ & $16(250)$ & $12(250)$ \\
\hline
\end{tabular}

${ }^{\mathrm{a}}$ zone of inhibition in $\mathrm{mm} ;{ }^{\mathrm{b}}$ Standard antibacterial drug.

\section{CONCLUSIONS}

In this work, the synthesis of naphthalene substituted Schiff base derivatives $\mathbf{4 a - m}$ derived from 2,2'-(naphthalene-2,7-diylbis(oxy))diacetohydrazide $\mathbf{3}$ with aromatic/hetero aldehyde in presence of acid catalyst under conventional heating and by the use of microwave irradiation, is reported. The advantages of the method employed include simple reaction set-up, high product yield, short reaction times and use of small amount of solvents or no solvents. The structures of the synthesized compounds have been confirmed through spectral characterization such as IR, ${ }^{1} \mathrm{H}$ NMR, ${ }^{13} \mathrm{C}$ NMR and mass spectra. From the antibacterial screening carried out, it is observed that compounds 4a-m exhibited activity which is comparable with activity of standard drug.

\section{ACKNOWLEDGEMENTS}

Authors gratefully acknowledge the management of VIT University, Vellore for encouraging and providing necessary facilities for carrying out this work.

\section{REFERENCES}

1. Sridhar, S.K.; Saravanan, M.; Ramesh, A. Eur. J. Med. Chem. 2001, 36, 615.

2. Mladenova, R.; Ignatova, M.; Manolova, N.; Petrova, T.; Rashkov, I. Eur. Polym. J. 2002, 38, 989.

3. Panneerselvam, P.; Nair, R.R.; Vijayalakshmi, G.; Subramanian, E.H.; Sridhar, S.K. Eur. J. Med. Chem. 2005, 40, 225.

4. Walsh, O.M.; Meegan, M.J.; Prendergast, R.M.; Nakib, T.A. Eur. J. Med. Chem. 1996, 31, 989.

5. Pandeya, S.N.; Sriram, D.; Nath, G.; Declercq, E. Eur. J. Pharmacol. 1999, 9, 25.

6. Pandeya, S.N.; Sriram, D.; Nath, D.; DeClercq, E. Pharm. Acta Helv. 1999, 74, 11.

7. Liu, M.C.; Lin, T.S.; Sartorelli, A.C. J. Med. Chem. 1992, 35, 3672.

8. Hodnett, E.M.; Dunn, J.W. J. Med. Chem. 1970, 13, 768. 
9. Valentina, P.; Ilango, K.; Deepthi, M.; Harusha, P.; Pavani, G. J. Pharm. Sci. Res. 2009, 1, 74.

10. Satyanarayana, V.S.V.; Madhumita, R.; Sivakumar, A. Asian J. Chem. 2010, 23, 1212.

11. Haviv, H.; Ratajczyk, J.D.; DeNet, R.W.; Kerdesky, F.A.; Walters, R.L.; Schmidt, S.P.; Holms, J.H.; Young, P.R.; Carter, G.W. J. Med. Chem. 1988, 31, 1719.

12. Patt, W.C.; Hamilton, H.W.; Taylor, M.D.; Ryan, M.J.; Taylor Jr., D.G.; Connolly, C.J.; Doherty, A.M.; Klutchko, S.R.; Sircar, I. J. Med. Chem. 1992, 35, 2562.

13. Bell, F.W.; Cantrell, A.S.; Hoegberg, M.; Jaskunas, S.R.; Johansson, N.G.; Jordan, C.L; Kinnick, M.D.; Lind, P.; Morin Jr., M.J. J. Med. Chem. 1995, 38, 4929.

14. Kumar, Y.; Green, R.; Wise, D.S.; Wotring, L.L.; Townsend, L.B. J. Med. Chem. 1993, 36, 3849.

15. Medime, E.; Capan, G. II Farmaco. 1994, 49, 449.

16. Metzger, J.V. Comprehensive Heterocyclic Chemistry I, Vol. 6, Pergamon: New York; 1984; p 234.

17. George, R.S.; Joseph, R.; George, K.E. Int. J. Polym Matter, 1993, 23, 17.

18. Chohan, Z.H.; Sherazi, S.K.A. Metal-Based Drugs, 1997, 4, 327.

19. Satyanarayana, V.S.V.; Sivakumar, A.; Ghosh, A.R. Letters in Drug Design and Discovery 2011, 8, 276.

20. Jin, L.; Chen, J.; Song, B.; Chen, Z.; Yang, S.; Li, Q.; Hu, D.; Xu, R. Bioorg. Med. Chem. Lett. 2006, 16, 5036.

21. Alcock, N.W.; Cook, D.F.; McKenzie, M.D.; Worthington, J.M. Inorg. Chem. Acta 1980, 38, 107.

22. Giguere, R.J.; Bray, T.L.; Duncan, S.M.; Majetich, G. Tetrahedron Lett. 1986, 27, 4945.

23. Loupy, A.; Petit, A.; Hamelin, J.; Texier-Boullet, F.; Jacquault, P.; Mathé, D. Synthesis 1998, 9, 1213.

24. Lindstrom, P.; Westman, J.; Lewis, A. Comb. Chem. High Throughput Screen. 2002, 5, 441.

25. Khanum, S.A.; Shashikanth, S.; Deepak, A.V. Bioorg. Chem. 2004, 32, 211. 